\title{
60 Jahre Tarifvertragsgesetz - Eine rechtspolitische Bilanz
}

60 Jahre ist das Tarifvertragsgesetz (TVG) in Kraft, ein Gesetz mit nur etwa einem halben Dutzend Paragrafen und wenigen Novellierungen in dieser langen Zeit. Das klingt nach einer Erfolgsgeschichte. Unter dem Strich handelt es sich auch um ein in hohem Maße gelungenes Gesetzgebungswerk, dessen Bedeutung seine „Väter“ kaum vorhersehen konnten. Der Beitrag zeichnet anhand ausgewählter Problemfelder des Tarifvertragsgesetzes die wichtigsten rechtspolitischen Diskussionslinien nach. Sie spiegeln zugleich im Zeitverlauf den Stand der jeweiligen tarifpolitischen Entwicklung wider.

\section{1 \\ Geburtsstunde des TVG - Konzeptionen und Kontroversen}

\subsection{STAATLICHE EINBINDUNG ODER LIBERALE AUSGESTALTUNG?}

Soweit ich im Folgenden die Geschichte des Tarifvertragsgesetzes - seine Entstehung nach Kriegsende zwischen 1946 und 1948 und sein Inkrafttreten am 9.4.1949, also zeitlich noch vor dem Grundgesetz (GG) vom 22.5.1949 - nachzeichne, wird sich dies auf einige wenige Grundzüge vor allem mit Blick auf die aktuelle Debatte beschränken (ausführlich: Nautz 1985, 1999; Herschel 1973).

Die grundsätzliche Kontroverse lässt sich wie folgt skizzieren: Die eine Konzeption wollte Tarifverträge in ein Modell von Wirtschaftsdemokratie „einbetten“. Dies wäre im Ergebnis auf eine stärkere staatliche Einbindung hinausgelaufen, wie beispielsweise die Überprüfung von Tarifinhalten durch staatliche Institutionen. Ein solcher Ansatz war mit den Namen Erich Potthoff und Wilhelm Herschel verbunden. ${ }^{1}$ Die andere Position befürwortete weitgehend staatsunabhängige, frei ausgehandelte Tarifverträge. Sie wurde nach zum Teil schwieriger Willensbildung von den Gewerkschaften und auch den Arbeitgeberverbänden vertreten. Hans Carl Nipperdey, ein hervorragender Jurist, ${ }^{2}$ brachte sie in die Form eines rechtlichen Entwurfs. Von beiden Alternativen setzte sich die liberale Ausgestaltung des Tarifrechts durch. Ent- scheidend hierfür war der Grundkonsens zwischen Gewerkschaften und der Arbeitgeberseite. Allerdings gab es damals bereits Kontroversen zwischen den Verbänden. Zwar wurden sie nicht konsequent ausgetragen, vor allem weil die Arbeitgeberverbände in den ersten Jahren nach Kriegsende die aus ihrer Sicht nützliche Kooperation mit der (organisierten) Arbeiterschaft nicht infrage stellen wollten (vgl. jedoch Kittner 2005, S. 660ff.). Die damaligen Dissenspunkte leben jedoch in der aktuellen Diskussion mit unterschiedlichem Gewicht wieder auf.

\subsection{KONTROVERSE THEMEN - EIN WETTERLEUCHTEN?}

Festzuhalten ist zunächst das Interesse der Arbeitgeber an Tarifverträgen für großräumige Tarifgebiete, also die Form des in jüngerer Vergangenheit vielfach und vehement kritisierten Flächentarifvertrages. Was das Verhältnis der beiden kollektiven Ebenen zueinander, Tarifvertrag und betriebliche Vereinbarung, betrifft, so war im Entwurf von Nipperdey zwar vorgesehen, dass Tarifverträge Vorrang vor Betriebsvereinbarungen hätten, sie sollten jedoch durch diese abgelöst werden können. Nach Intervention der Gewerkschaften wurde die Vorrangproblematik, die in der aktuellen Debatte eine so zentrale Bedeutung gewonnen hat, klar zugunsten des Tarifvertrages gelöst. Auf ihren Einspruch hin schloss man eine Ablösung der Tarifnormen durch Betriebsvereinbarungen aus. Kritisch äuBerte sich ein Teil der Arbeitgeber zu einem Ausbau der normativen Wirkung des Tarifvertrages durch die Elemente „Verzicht auf
Tarifrechte nur durch die Tarifparteien selbst“ und „die Nachwirkung der tariflichen Normen". All diese und weitere, eher untergeordnete Streitpunkte stellte die Arbeitgeberseite jedoch zurück. Damit konnte der modifizierte Entwurf Nipperdeys im Wirtschaftsrat, dem Wirtschaftsparlament des vereinigten Wirtschaftsgebiets, im November 1948 einstimmig verabschiedet werden. Das Tarifvertragsgesetz trat am 9.4.1949 in Kraft.

Das Gesetz, das lediglich einen Rahmen setzt - allerdings mit einigen scharf ausgeprägten Grundprinzipien (Tarifinhalte, Tarifparteien, Tarifgebundenheit, normative Wirkung) -, war so offen konzipiert, dass es bislang alle Veränderungen der gesellschaftlichen und tarifpolitischen Koordinaten unbeschadet überstand. Die wichtigste Änderung war die Einführung des

\footnotetext{
Potthoff war einer der beiden Leiter des Wirtschaftswissenschaftlichen Instituts der Gewerkschaften (WWI). Herschel war in maßgeblicher Position in der Arbeitsverwaltung tätig, zunächst in der britischen, dann in der Bizone.

2 Nipperdey wurde später der erste Präsident des Bundesarbeitsgerichtes (BAG) und war in geschmeidiger Anpassung an die jeweiligen Machtverhältnisse in den ersten Jahren nach dem Krieg kurzfristig auch Berater des Deutschen Gewerkschaftsbundes (DGB); zu ihm jüngst Adomeit 2006, S. 745ff.; hierzu ergänzend Arbeit und Recht 2006, S. 359.
}

Ulrich Zachert, Prof. Dr., Universität Hamburg, Department für Wirtschaft und Politik. Arbeitsschwerpunkte: Arbeitsrecht, Europäisches Recht, Rechtsvergleichung. e-mail: Ulrichzachert@aol.com 
$\$ 12$ a im Jahre 1974, durch den Tarifverträge auch auf arbeitnehmerähnliche Personen erstreckt werden konnten, was besonders im Bereich der Medien praktische Bedeutung erlangte. Allerdings sollte in diesem Zusammenhang nicht aus den Augen verloren werden, dass Änderungen anderer Gesetze in „benachbarten Regelungsbereichen" Auswirkungen unterschiedlicher Art auf die Machtbalance der Tarifparteien hatten. Hierzu gehört neben dem bislang folgenlosen „Gesetz über Mindestarbeitsbedingungen" von $1952^{3}$ die Neufassung des $\$ 116$ AFG (jetzt 146 SGB III) im Jahr 1985 (Problem der „kalten Aussperrung“) sowie das Inkraftsetzen des Arbeitnehmerentsendegesetzes im Jahr 1996 mit diversen Novellierungen in den Folgejahren („tariflich gestützter Mindestlohn in festgelegten Branchen").

\subsection{DER VERFASSUNGSRECHTLICHE "ÜBERBAU“}

Erst später kam das Verhältnis des Tarifvertrages zum Grundgesetz genauer ins Blickfeld. Letzteres war zunächst als Provisorium gedacht, erwies sich jedoch bis heute - auch fast 20 Jahre nach der deutsch-deutschen Einigung - als dauerhafter normativer Bauplan. Der einschlägige Art. 9 Abs. 3 GG (Koalitionsfreiheit) ist in seinem materiellen Gehalt recht blass. Er enthält weder eine ausdrückliche Gewährleistung der Tarifautonomie noch des Streikrechts. Im Hinblick auf die Tarifautonomie gingen die „Väter und (wenigen) Mütter" der Verfassung davon aus, diese noch aus der Weimarer Zeit als gefestigt voraussetzen zu können (Verordnung über Tarifverträge von 1918 und Art. 159, 165 Weimarer Verfassung von 1919). Das erwies sich als zutreffend und wurde vom Bundesverfassungsgericht bereits in frühen Entscheidungen bestätigt (BVerfG 18. 11. 1954; BVerfGE 4, 96). Allerdings stellte es sich in den Folgejahren als erhebliche Herausforderung für die Rechtsprechung des Bundesverfassungs- und Bundesarbeitsgerichts dar, in dem Zusammenspiel von Tarifvertrag und Grundgesetz Umfang und Grenzen der Tarifautonomie im Einzelfall auszuloten. Hier stand sie vor der Aufgabe, im Arbeitskampfrecht, aber auch im Tarifrecht, eine Kasuistik zu entwickeln, die inzwischen eine recht feinmaschige Dimension angenommen hat (Dieterich 2008, S. 71, 73ff.).

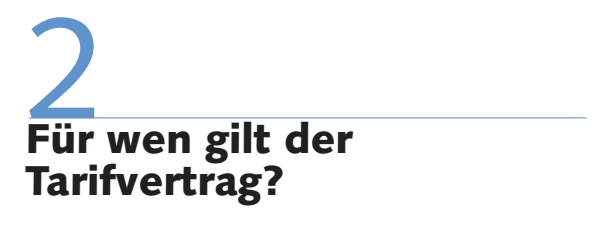

\subsection{EINGESPIELTE TARIFPRAXIS IN DEN 1960ER JAHREN - ZWEI KONFLIKTFELDER}

In den 1950er und 1960er Jahren, die durch ein hohes Wirtschaftswachstum gekennzeichnet waren („Wirtschaftswunder"), sind keine spektakulären rechtspolitischen Kontroversen um das Tarifvertragsgesetz zu verzeichnen. ${ }^{4}$ Zwar hatte sich die mit dem Gedanken der sogenannten „aktiven Tarifpolitik" verbundene Erwartung nicht erfüllt, über die Tarifpolitik die Einkommensverhältnisse zwischen Selbstständigen und abhängig Beschäftigten zugunsten Letzterer zu verändern. Jedoch trug die Tarifpolitik insgesamt dazu bei, den Zuwachs an volkswirtschaftlichen Werten zwischen Arbeitnehmern und Selbstständigen ungefähr gleich zu verteilen, und sie wirkte so einem Abbau der Lohnquote entgegen.

Von dem relativ geräuschlosen Umgang mit dem rechtlichen Rahmen des TVG in der gut eingespielten Tarifpraxis gab es allerdings zwei Ausnahmen. Sie betrafen den Geltungsbereich bzw. die Bindung des Tarifvertrages in ganz unterschiedlichen Sachverhaltskonstellationen. Zum einen bemühten sich einige DGB-Gewerkschaften, durch verbindliche Differenzierungsklauseln in Tarifverträgen begrenzte tarifliche Leistungen, $z$. B. ein leicht erhöhtes Urlaubsgeld, mit unterschiedlichen rechtlichen Instrumenten (Spannensicherungs- oder Tarifausschlussklauseln) ausschließlich ihren Mitgliedern zukommen zu lassen. Zum anderen stieß die Allgemeinverbindlicherklärung (AVE) des $₫ 5$ TVG, eine der wenigen Neuerungen des TVG gegenüber seinem Vorläufer, der Tarifvertragsordnung vom 23.12. 1918, auf den Widerstand einzelner Arbeitgeber.

\subsection{SONDERREGELUNGEN FÜR GEWERKSCHAFTSMITGLIEDER}

Der Versuch der damaligen IG Textil, im Tarifvertrag rechtlich verbindlich ein zusätzliches Urlaubsgeld in Höhe von 40 DM bis 60 DM für ihre Mitglieder abzusichern, führte Mitte der 1960er Jahre zu einer Grundsatzentscheidung des Großen Senats des BAG (BAG GS 29.11.1967, AP Nr. 13 zu
Art. 9 GG) und zu einer lebhaften Diskussion in der Rechtslehre. Auch der 46. Deutsche Juristentag beschäftigte sich 1966 mit dieser Frage (aus der Kommentarliteratur nur Kempen/Zachert/Wendeling-Schröder TVG $\$ 4$ Rn. 238ff. mit Nachw.). Das BAG erklärte diese Form der verbindlichen Differenzierung für rechtswidrig, weil mit derartigen Regelungen den Außenseitern eine Art Ausgleichsleistung für die Inanspruchnahme gewerkschaftlicher Arbeiten auferlegt wird. Dies müsse bei ihnen zwangsläufig das Gerechtigkeitsempfinden verletzen. Ferner werde das in Art. 9 Abs. 3 GG gewährleistete Grundrecht der negativen Koalitionsfreiheit verletzt, da die fragliche Differenzierungsklausel einen sozial inadäquaten Druck auf die Außenseiter ausübe, den sie nicht hinzunehmen bräuchten. Diese Argumentation des Großen Senats erscheint konstruiert und unausgewogen. Unter anderem spielt in den Erwägungen des Gerichts das „Gerechtigkeitsempfinden der Gewerkschaftsmitglieder" keine Rolle, die durch ihre Gewerkschaftsbeiträge die Arbeitsbedingungen der Außenseiter faktisch mitfinanzieren. Jüngst deutete das BAG an, dass es von der Linie, die der Große Senat vorgezeichnet hat, wohl abweichen werde (BAG 9.5.2007, NZA 2007, 1439 Rn. 30f; auch LAG Niedersachsen 11.12.2007, DB 2008 1977; dazu Ulber/Strauß 2008, S. 1970ff.).

Die tarifpolitische Diskussion in den DGB-Gewerkschaften über das Pro und Kontra des Abschlusses von Differenzierungsklauseln wurde in den 1970er Jahren sehr fundamentalistisch geführt. Kleinere Gewerkschaften in eher mittelständisch strukturierten Branchen praktizierten sie trotz des Verdikts der höchstrichterlichen Rechtsprechung, soweit sie auf kooperationsbereite Arbeitgeber trafen. Große Gewerkschaften, wie die IG Metall und Gewerkschaft öffentliche Dienste, Transport und Verkehr (ÖTV), lehnten sie strikt ab, vor allem weil sie um ihren Anspruch auf Repräsentation aller Arbeitnehmer, also nicht nur ihrer Mitglieder, fürchteten (ausführlich Seitenzahl et al. 1976). Vor dem Hintergrund signifikanter Mitgliederverluste sind derartige Tarifstrategien aktuell auch bei großen Gewerkschaften kein Tabubruch mehr. Dass die Bäume verbindlicher Vorteilsregelungen auch in Zukunft

\footnotetext{
3 Zur neusten Entwicklung vgl. die Abschnitte 2.3 und 4.

4 Zu den Arbeitskämpfen und zum Arbeitskampf-
} recht in dieser Zeit vgl. Kittner 2005, S. $602 \mathrm{ff}$. 
nicht in den Himmel wachsen, dafür sorgt schon, dass sie ohne Außenseiter nicht erstreikt werden können (Gamillscheg 1967, S. 45,53$)$.

\subsection{AUSWEITUNG DES TARIFLICHEN GELTUNGSBEREICHS}

Nahezu spiegelverkehrt zu dem Aspekt tariflicher Differenzierungsklauseln geht es bei der Allgemeinverbindlicherklärung (AVE) um die Ausweitung des tariflichen Geltungsbereichs unter bestimmten, $\operatorname{im} \$ 5$ TVG definierten Voraussetzungen. Die AVE wurde lange Zeit von den Tarifparteien vor allem in mittelständisch geprägten Branchen im Konsens praktiziert. Sie wurde erst im Zuge massiver Kritik am Flächentarifvertrag - als Folge von Tendenzen einer Deregulierungs- und Flexibilisierungspolitik in Staat und Gesellschaft - infrage gestellt (Kirsch 2003, S. 405ff.). Jedoch deuteten sich bereits in den 1970er Jahren Sollbruchstellen des Grundkonsenses zwischen den Tarifparteien zu dieser Thematik an. Das Bundesverfassungsgericht (BVerfG) hatte darüber zu entscheiden, ob ein Außenseiter, der nicht Mitglied des Arbeitgeberverbandes war, durch die Allgemeinverbindlicherklärung gezwungen werden könne, die tariflichen Normen anzuwenden (BVerfG 24.5.1977, BVerfGE 44, 322). Das Gericht bestätigte dies. $₫ 5$ TVG verstoße nicht gegen die Verfassung. Insbesondere in Zeiten schlechter Konjunktur könnten Arbeitgeber veranlasst sein, sich durch Einstellung von Arbeitnehmern zu untertariflichen Löhnen Vorteile gegenüber der Konkurrenz zu verschaffen. Der Sinn der AVE bestehe darin, einer solchen „Lohndrückerei“ und „Schmutzkonkurrenz" entgegenzuwirken. Bei der Allgemeinverbindlicherklärung handele es sich um eine Kombination von autonomem Recht der Tarifparteien, dessen Legitimation sich aus Art. 9 Abs. 3 GG ergebe, und staatlichem Einfluss, dessen Grundlage das Demokratieprinzip des Art. 20 GG sei. Eine Verletzung der sogenannten negativen Koalitionsfreiheit liege nicht vor. Ein lediglich mittelbarer Druck auf den Außenseiter, sich der Koalition anzuschließen, müsse hingenommen werden.

Welche Tragweite diese Entscheidung hatte, sollte sich erweisen, als die abnehmende Bindungsfähigkeit des Flächentarifvertrages seit den 1990er Jahren zu Initiativen führte, auf der Grundlage des Arbeitnehmer-Entsendegesetzes (AEntG) von
1996 gesetzlich gestützte Tariflöhne einzuführen. Die heftige Kritik hieran von Teilen der Arbeitgeberseite und der Rechtswissenschaft ${ }^{5}$ gipfelte wiederum in einer Vorlage beim Bundesverfassungsgericht. Das Gericht hat sich in der Entscheidung im Juli 2000 (BVerfG 18.7.2000 - Kammer - AP Nr. 4 zu $\$ 1$ AEntG) auf seinen Beschluss aus dem Jahr 1977 bezogen und mit inhaltlich gleichen Erwägungen betont, dass auch die Festlegung von Mindestentgelten durch staatliche Verordnung nicht gegen die negative Koalitionsfreiheit von Arbeitgebern nach Art. 9 Abs. 3 GG verstoße, die nicht Mitglied im zuständigen Verband sind. Entsprechend argumentiert das Bundesverfassungsgericht in einer Verfassungsbeschwerde gegen ein Tariftreuegesetz (vgl. Schulten/ Pawicki 2008, S. 184ff.), nach dem öffentliche Aufträge vor allem im Baubereich nur an solche Arbeitgeber vergeben werden dürfen, die die tariflichen Bedingungen einhalten (BVerfG 1.7.2006, BVerfG E 111, 202).

Alle diese höchstrichterlichen Urteile sind nur die Spitze eines Eisbergs. Sie dokumentieren heftigen Widerstand gegen das Setzen von Mindeststandards im Bereich der Lohn- und Arbeitsbedingungen, die weiter reichen als die „klassischen Tarifvereinbarungen" mit begrenzter Tarifbindung nur für Verbandsmitglieder. Die Rechtsprechung des Bundesverfassungsgerichts ist dieser Linie nicht gefolgt. Allerdings hat sich die Debatte nunmehr auf die europäische Ebene verlagert. Die Niederlassungs- (Art. 43 EGV) und Dienstleistungsfreiheit ( $\$ 49 \mathrm{EGV}$ ) des EU-Vertrages wird zunehmend erfolgreich gegen den nach nationalem Recht gewährleisteten Schutzbereich von Tarifautonomie und Arbeitskampfrecht in Stellung gebracht (EuGH 3.4.2008 - Rüffert, NZA 2008, 537; EuGH 11.12.2007 - Viking, NZA 2008, 124; EuGH 18.12.2007 - Laval, AuR 2008, 59).

\section{3 \\ Tarifvertrag in Konkurrenz zu anderen Regelungs- ebenen}

\subsection{TARIFVERTRAG UND ARBEITS- VERTRAG - DAS GÜNSTIGKEITS- PRINZIP ALS ALLZWECKWAFFE GEGEN ARBEITSZEITVERKÜRZUNG?}

Mitte der 1980er Jahre wurde dem Günstigkeitsprinzip des $₫ 4$ Abs. 3 TVG teilweise eine Dimension zugemessen, die über die traditionelle Auslegung weit hinausgeht. $\$ 4$ Abs. 3 TVG räumt (nur) günstigeren arbeitsvertraglichen Vereinbarungen Vorrang vor tariflichen Normen ein. Der Streit entzündete sich um die Frage, was eine günstigere Regelung sei. Ist es z. B. günstiger, im Arbeitsvertrag eine längere Arbeitszeit als die tarifliche, verbunden mit einer begrenzten Beschäftigungssicherung $\mathrm{zu}$ vereinbaren? Gesellschaftspolitischer Hintergrund der Kontroverse war vor allem die schrittweise Einführung der 35-StundenWoche durch Tarifvertrag in den Jahren 1984, 1987 und 1990. Sie stieß nach der harten Auseinandersetzung des Jahres 1984 mit Flächenstreiks, kalten und heißen Aussperrungen und dem sogenannten „LeberKompromiss" als Ergebnis einer schwierigen Schlichtung auf Grenzen der Akzeptanz auf Arbeitgeberseite. ${ }^{6}$ Eine Auslegung, die individuellen Regelungen Vorrang vor tariflichen einräumt, würde zumindest eine partielle Korrektur der Tarifverträge zur wöchentlichen Arbeitszeitverkürzung ermöglichen (hierzu mit weiteren Überlegungen und Nachweisen Kempen/Zachert 2006, § 4 TVG Rn. 229ff.).

Abgesehen von existenziellen ökonomischen und politischen Krisenzeiten hat sich die Tariflandschaft in keinem Zeitraum radikaler verändert als in den Jahren seit dem sogenannten „Leber-Kompromiss“ zur 35-Stunden-Woche von 1984 mit Öffnungen für differenzierte Lösungen der Arbeitszeitdauer (bestätigt durch BAG 18.8.1987, AP Nr. 23 zu $\$ 77$ BetrVG 1972). Das betrifft vor allem die Tendenz der Verlagerung von Kompetenzen vom (Flächen-) Tarifvertrag auf die betriebliche und mit Einschränkungen auf die arbeitsvertragliche Ebene. Inzwischen sind die Tarifverträge zu zahlreichen Themenfeldern auch über die Arbeitszeit hinaus so weit und vielfältig geöffnet, dass dies bereits ungelöste Probleme im Hinblick auf die Steuerungsfähigkeit des „Gesamtinstruments Tarifvertrag" aufwirft (Bispinck et al. 2007, S. 15ff.; empirisch Oppolzer/ Zachert 2000; Höland et al. 2000).

5 Zum Streitstand vgl. ausführlich bei Däubler/Lakies 2006 Anhang 2 zu § 5 TVG Rn. 54ff.; Peter et al. 2004, S. $126 \mathrm{ff}$.

6 In diesem Jahr erschien auch der erste grundlegende rechtliche Beitrag zu diesem Thema, vgl. Joost 1984, S. 173; zu den um die Arbeitszeitverkürzung geführten Arbeitskämpfen und zum Arbeitskampfrecht in dieser Zeit vgl. Kittner 2005, S. $206 \mathrm{ff}$ 
Gleichwohl hat die Kontroverse um die Auslegung des Günstigkeitsprinzips an Schärfe kaum abgenommen. Die Positionen, welche die Rechtslehre zum Günstigkeitsvergleich vorschlägt, sind inzwischen Legion. Gemeinsames Ziel oder jedenfalls Ergebnis der unterschiedlichen methodischen Ansätze eines neuen Vergleichsmaßstabs des Günstigkeitsprinzips ist es vor allem, beschäftigungssichernde Zusagen in den Günstigkeitsvergleich mit einzubeziehen. Die zwingende Wirkung des Tarifvertrages soll aufgelockert werden, weil sich diese beschäftigungsfeindlich auswirke (so z. B. Rieble 2004, S. 31). Das Bundesarbeitsgericht ist dieser weiten Auslegung des Günstigkeitsvergleichs nicht gefolgt. Man könne nur das miteinander vergleichen, was sachlich zusammengehöre, also z. B. die tarifliche Arbeitszeit mit der arbeitsvertraglichen Arbeitszeit. Alles andere sei eine tarifpolitische Entscheidung der Parteien, in die die Gerichte nicht einzugreifen hätten (BAG 20.4.1999, AP Nr. 98 zu Art. 9 GG).

Letztlich geht es hier um die Grundsatzfrage, ob der zwingende Charakter des Tarifvertrages, der den tarifgebundenen Arbeitnehmern eine Mindestniveaugarantie im Sinne sicherer Mindestbedingungen gewährleistet, durch das tarifliche Günstigkeitsprinzip infrage gestellt werden darf. Hätte man anders entschieden als das Bundesarbeitsgericht, würde der Tarifvertrag seine zwingende Wirkung verlieren. Es würde ein Regelungssystem gelten, in dem der Tarifvertrag lediglich noch eine Leitlinie („gentleman's agreement“) darstellt. Dies entspricht der angloamerikanischen Rechtskultur. Sie folgt jedoch anderen Traditionen als denen, die die meisten Länder Kontinentaleuropas prägen (Zachert 2007, S. $1111 \mathrm{ff}$.).

\subsection{TARIFVERTRAG UND BETRIEBSVEREINBARUNG - DAS GÜNSTIGKEITSPRINZIP ALS MITTEL ZUR UMKEHRUNG DES RANGVERHÄLTNISSES?}

Vor dem Hintergrund der angesprochenen Probleme prägt bis heute die Kontroverse um das Günstigkeitsprinzip die rechtspolitische und rechtliche Diskussion. Dabei geht es nicht nur um das Verhältnis des Tarifvertrages zum Arbeitsvertrag, sondern vor allem um das Rangverhältnis des Tarifvertrages zur Betriebsvereinbarung. Die rechtliche Kontroverse überrascht, denn anders als $₫ 4$ Abs. 3 TVG legt die hier einschlägige Spezialvorschrift des $\$ 77$ Abs. 3 BetrVG einen klaren Vorrang des Tarifvertrages vor Betriebsvereinbarungen fest. Eine Ausnahme durch Günstigkeitserwägungen ist im Gesetz nicht vorgesehen. Gleichwohl hat es auch hier unzählige Publikationen gegeben, die Argumente zusammentragen, um mithilfe des Günstigkeitsprinzips das Rangverhältnis zwischen Tarifvertrag und Betriebsvereinbarung umzukehren (vgl. Löwisch/Rieble $2004 \$ 4$ Rn. 260ff. mit Nachweisen.).

Diesen Weg ist die Rechtsprechung zu Recht ebenfalls nicht mitgegangen (BAG 24.2.1987, AP Nr. 21 zu $\$ 77$ BetrVG 1972; ArbG Marburg, AuR 1996, 46). Eine Verdrängung der Tarifparteien oder die Reduzierung ihrer Aufgaben auf eine bloße „Abnickfunktion" der tarifändernden Vereinbarungen, auf die sich die Betriebsparteien geeinigt haben, entspricht nicht ihrem verfassungsrechtlichen Gestaltungsauftrag nach Art. 9 Abs. 3 GG. Den Betriebsparteien in ihrer engeren, auf den Betrieb bezogenen Legitimation ist es untersagt, ein kollektives Instrument wie den Arbeitskampf zur Konfliktlösung einzusetzen. Das drückt das Verbot des $\$ 74$ Abs. 2 BetrVG deutlich aus. Damit soll verhindert werden, dass Nichtmitglieder über Betriebsvereinbarungen die tariflichen Rechtspositionen der Gewerkschaftsmitglieder beeinflussen und so letztlich die Gestaltungsaufgaben der Tarifpolitik konterkarieren können. Auch dies widerspräche dem freiheitlichen Charakter der Tarifautonomie des Art. 9 Abs. 3 GG.

\section{3 „BETRIEBLICHE BÜNDNISSE FÜR ARBEIT“ ${ }^{\prime}$ DURCH GESETZLICHE ÖFFNUNGSKLAUSELN?}

Nachdem die Rechtsprechung einem Umbau der gewachsenen Tarifstrukturen einen Riegel vorgeschoben hatte, trat die Politik auf den Plan. Den Stein ins Wasser warfen die Unionsparteien mit dem Gesetzesentwurf zur sogenannten Modernisierung des Arbeitsrechts (ArbRModG) vom 18.6.2003 (BT Drucks. 15/1182 vom 18.6.2003). Vor allem im Wahlkampf des Sommers und Herbstes 2005 wurden die entsprechenden Vorschläge mehrfach aufgegriffen und der Öffentlichkeit vorgestellt. Der ausformulierte Entwurf sieht zum einen eine Umwertung des Günstigkeitsprinzips des $₫ 4$ Abs. 3 TVG vor. Im Verhältnis des Tarifvertrages zum Arbeitsvertrag sollen beim
Günstigkeitsvergleich Beschäftigungsaussichten mitberücksichtigt werden. Eine Abweichung gelte als günstiger, wenn Betriebsrat und zwei Drittel der Belegschaft dem zustimmen. Zum anderen wird im Verhältnis des Tarifvertrages zur Betriebsvereinbarung der nach $\$ 77$ Abs. 3 BetrVG bestehende Vorrang des Tarifvertrags gegenüber betrieblichen Vereinbarungen ebenfalls in sein Gegenteil verkehrt. Ein „Betriebliches Bündnis für Arbeit“ soll möglich sein, wenn mindestens zwei Drittel der Belegschaft zustimmen, die Vereinbarung den Tarifparteien angezeigt wird und diese nicht begründet widersprechen.

Dies stieß auf verfassungsrechtliche Bedenken in der Rechtswissenschaft. Es läge ein Verstoß gegen die zwingende (normative) Wirkung des Tarifvertrages vor. $\mathrm{Zu}-$ dem werde der Grundsatz der positiven Koalitionsfreiheit der Gewerkschaften und die durch Art. 9 Abs. 3 Satz 2 GG geschützte Individualposition der Gewerkschaftsmitglieder sowie das vertragskonstituierende Paritätsprinzip als Voraussetzung für „die Richtigkeitsgewähr von Verträgen“ verletzt (Dieterich 2002, S. 1ff.; Zachert 2004, S. 123ff.). Die damals aktuellen Pläne werden zurzeit nicht ernsthaft diskutiert, da sie nicht Teil des Koalitionsvertrages zwischen CDU und SPD im Oktober des Jahres 2005 geworden sind. Ob sie wirklich aufgegeben wurden und ob sich die „Entdeckung der Tarifautonomie" durch die Kanzlerin ${ }^{7}$ und manchen Vertreter in der Rechtswissenschaft, der vorher ebenfalls eine entschieden gegenteilige Auffassung vertrat (Sodan 2008; dazu Zachert 2008), lediglich auf die Abwehr von gesetzlichen Mindestlöhnen beschränkt, wird von den gesellschaftlichen Koordinaten der nächsten Jahre abhängen.

\section{1}

\section{Ausblick}

Dies führt abschließend zu der Frage, wohin die Reise des Tarifvertragsgesetzes gehen könnte, nachdem es - bei allen Kontroversen - 60 Jahre einen belastbaren Rahmen für die Tarifpolitik der Verbände abgegeben hat. Drei Problembereiche stehen aus meiner Sicht auf der Tagesordnung.

\footnotetext{
So formulierte beispielsweise das Handelsblatt vom 19.6.2008: "Merkel mahnt Vorrang für Tarifverträge an".
} 
Zum einen ergeben sich Risiken für die Tarifstrukturen, weil bei den Akteuren der Tarifpolitik bereits seit Langem Anzeichen einer gewissen Schwäche festzustellen sind. Namentlich die Geschäftsgrundlage gewerkschaftlicher Arbeit hat sich geändert. Die Einheitsgewerkschaft, die auch für den schwierigen Ausgleich der Interessen der Arbeitnehmer in den eigenen Reihen steht, hat an Bindungsfähigkeit verloren. Entsprechendes gilt für den Flächentarifvertrag mit seiner Friedenspflicht, dem geordneten Verfahren nach seinem Ablauf, insbesondere den durchweg vereinbarten tariflichen Schlichtungsverfahren. Die DGB-Gewerkschaften sehen sich seit einigen Jahren einerseits einer Unterbietungskonkurrenz durch kleine, oft Pseudogewerkschaften ausgesetzt. ${ }^{8}$ Andererseits sind sie mit einer Überbietungskonkurrenz von Spezialistengewerkschaften konfrontiert, denen es gelingt, die Interessen ihrer Klientel zum Teil mit beachtlicher Militanz durchzusetzen (Berg et al. 2008, AKR Rn. 234aff.; vgl. hierzu auch Dribbusch in diesem Heft). In der Rechtswissenschaft hat dies zu Vorschlägen geführt, ein Schlichtungsverfahren verbindlich durchzuführen, um dem Gedanken der Tarifeinheit Rechnung zu tragen. ${ }^{9}$ In diesem Zusammenhang verdient Erwähnung, dass die Mitgliedsgewerkschaften des DGB durch ein autonomes verbandsinternes Verfahren „in ihren Reihen“ die Tarifeinheit herstellen. Dies ist bei allen praktischen Schwierigkeiten ein beachtlicher Beitrag zur Gewährleistung transparenter Tarifstrukturen. Die DBG-Schiedsgerichtsbarkeit wird von der höchstrichterlichen Rechtsprechung anerkannt, steht jedoch (zunehmend) in der Kritik der
Rechtswissenschaft (Nachweise nur ErfK/ Dieterich 2009, Art. 9 GG Rn. 68 und ErfK/ Franzen $₫ 2$ TVG Rn. 35 mit unterschiedlichen Positionen).

Zweitens wird der bereits beschrittene Weg, die Festlegung von Mindestlöhnen stärker in staatliche Verantwortung zu legen (jüngst: Bispinck/Schulten 2008, S. 151ff.), Fragen der Abstimmung der jeweiligen Regelungsebenen, also zwischen tariflich und staatlich definiertem Mindestlohn, aufwerfen. Die rechtlichen Probleme sind aus meiner Sicht lösbar. Insbesondere sprechen Erfahrungen mit gesetzlichen Mindeststandards bei der Arbeitszeit (Arbeitszeitgesetz) und beim Urlaub (Bundesurlaubsgesetz) dagegen, dass ein gesetzlicher Mindestlohn, der nur eine Untergrenze setzt, die Tarifautonomie schwächt. Das korrespondiert mit Erfahrungen in unseren europäischen Nachbarstaaten, die ganz überwiegend beides haben: Mindestlohngesetze und eine funktionierende Tarifautonomie (Schulten et al. 2006). Rechtliche Stellungnahmen, die bereits in den aktuellen Gesetzesinitiativen, der Erweiterung des Arbeitnehmer-Entsendegesetzes auf andere Branchen und der Konkretisierung des „Gesetzes über die Festsetzung von Mindestarbeitsbedingungen" aus dem Jahr 1952, einen Verstoß gegen die Tarifautonomie (Art. 9 Abs. 3 GG) sehen (vgl. Abschnitt 3.3.), deuten jedoch möglicherweise ein Wetterleuchten an, dem weitere Auseinandersetzungen folgen könnten (vgl. als Überblick: Dieterich 2008, S. 71, 79ff.).

Drittens komme ich noch einmal auf die im Abschnitt 2.2 bereits angesprochene europäische Ebene zurück. Die europäischen Vertragswerke, vor allem der EU-
Vertrag, enthalten erhebliche Interpretationsspielräume bei der Abwägung der ausdrücklich geschützten Niederlassungs(Art. 43 EGV) und Dienstleistungsfreiheit (Art. $49 \mathrm{EGV}$ ) gegenüber der dort eher blass konturierten Tarifautonomie und dem Arbeitskampfrecht (Art. 136 EGV). Nach den jüngsten Entscheidungen des EuGH (zu den Fällen Rüffert, Vinking, Laval) scheint sich die Waage in Richtung eines Vorrangs von Marktfreiheit gegenüber einem sozialen Mindestschutz zu neigen. Das steht im Widerspruch zu den Grundlinien der Rechtsprechung des Bundesverfassungsgerichts. Nach ihr wird die typischerweise bestehende strukturelle Unterlegenheit des einzelnen Arbeitnehmers beim Aushandeln von Arbeitsverträgen durch die Tarifautonomie ausgeglichen. Ihr kommt ein hoher Stellenwert zu, da (nur) sie darauf angelegt ist, ein annähernd gleichgewichtiges Aushandeln der Lohnund Arbeitsbedingungen zu ermöglichen (BVerfG 20.6.1991, BVerfGE 84, 212, 229).

Vielleicht stellt es sich bald als nationale Aufgabe, die über 60 Jahre gewachsenen Tarifstrukturen und ihren konkreten Rahmen, das Tarifvertragsgesetz, gegenüber marktliberalen Zugriffen aus Europa zu verteidigen (ähnlich jüngst Däubler 2008, S. 415).

8 Zu den Rechtsproblemen bei einem tariflich gestützten Mindestlohn vgl. Bieback et al. 2007 als Replik auf Thüsing/Lembke 2007, S. 87ff.

9 Hromadka (2008, S. 384ff.) schlägt hierfür eine Änderung des TVG vor; Kamanabrou (2008, S. $241 \mathrm{ff}$.) fokussiert auf richterrechtliche Gestaltung; kritisch hierzu: Zachert (2009, S. 23, 26f.). 


\section{LITERATUR}

Achten, U. (Hrsg.) (2007): Flächentarifvertrag und betriebsnahe Tarifpolitik, Hamburg

Adomeit, K. (2006): Hans Carl Nipperdey als Anreger für eine Neubegründung des juristischen Denkens, in: Juristenzeitung 15/16, S. 745-751 Berg,P./Platow,H./Schoof,Chr./Unterhinninghofen,H. (2008): Tarifvertragsgesetz und Arbeitskampfrecht, Frankfurt/M Bieback, K.-J./Dieterich, Th./Hanau, P./Kocher, E./Schäfer, C. (2007): Tarifgestützte Mindestlöhne, Baden-Baden

Bispinck, R./Schulten, Th. (2008): Aktuelle Mindestlohndebatte: Branchenlösungen oder gesetzlicher Mindestlohn?, in: WSI-Mitteilungen 3,

S. 151-157

Bispinck, R./Schulz, H./Wagner, A. (2007): Flächentarifvertrag im Wandel, in: Achten, U. (Hrsg.): Flächentarifvertrag und betriebsnahe Tarifpolitik, Hamburg, S. 15-24

Däubler, W. (2008): ITF- Aktionen gegen Billig-Flaggen-Schiffe - im Widerspruch zum EG-Recht?, in: Arbeit und Recht 12 , S. 409-417

Däubler, W. (Hrsg.) (2006): Tarifvertragsgesetz, Baden-Baden

Dieterich, Th. (2002): Flexibilisiertes Tarifrecht und Grundgesetz,

in: Recht der Arbeit 1, S. 1-17

Dieterich, Th. (2008): Tarifautonomie: Altes Modell - neue Realität,

in: Kritische Justiz 1, S. 71-81

Erfurter Kommentar zum Arbeitsrecht (2009), München

Gamillscheg, F. (1967): Nochmals: Zur Differenzierung nach der Gewerkschaftszugehörigkeit, in: Betriebsberater 3, S. 45-53

Herschel, W. (1973): Zur Entstehung des Tarifvertragsgesetzes, in: Zeitschrift für Arbeitsrecht 2, S. 183-200

Höland, A./Reim, U./Brecht, H. (2000): Flächentarifvertrag und Günstigkeitsprinzip, Baden-Baden

Hromadka, W. (2008): Entwurf eines Gesetzes zur Regelung der Tarifkollision, in: Neue Zeitschrift für Arbeitsrecht 7, S. 384-391

Joost, D. (1984): Tarifliche Grenzen der Verkürzung der Wochenarbeitszeit, in: Zeitschrift für Arbeitsrecht 2, S. 173-203

Kamanabrou, S. (2008): Der Streik durch Spartengewerkschaften - Zulässigkeit und Grenzen, in: Zeitschrift für Arbeitsrecht 2, S. 241-281

Kempen, O. E./Zachert, U. (Hrsg.) (2006): Tarifvertragsgesetz, Frankfurt/M.

Kirsch, J. (2003): Die Allgemeinverbindlichkeit von Tarifverträgen ein Instrument in der Krise, in: WSI-Mitteilungen 7, S. 405-412
Kittner, M. (2005): Arbeitskampf, München

Löwisch, M./Rieble, V. (2004): Tarifvertragsgesetz, München

Nautz, J. (1985): Die Durchsetzung der Tarifautonomie in Westdeutschland, Frankfurt M./Bern/New York/Nancy

Nautz, J. (1999): Die Entstehung des Tarifvertragsgesetzes, in: WSI-Mitteilungen 7, S. 437-444

Oppolzer, A./Zachert, U. (2000): Krise und Zukunft des Flächentarifvertrages, Baden-Baden

Peter, G./Kempen, O. E./Zachert, U. (2004): Die Sicherung tariflicher Mindeststandards, Baden-Baden

Rieble, V. (2004): Tarifvertrag und Beschäftigung, in: Zeitschrift für Arbeitsrecht 1, S. 1-65

Schulten,Th./Bispinck,R./Schäfer,C. (Hrsg.) (2006): Mindestlöhne in Europa, Hamburg

Schulten, Th./Pawicki, M. (2008): Tariftreueregelungen in Deutschland Ein aktueller Überblick, in: WSI-Mitteilungen 4, S. 184-190

Seitenzahl, R./Zachert, U./Pütz, H.-D. (1976): Vorteilsregelungen für Gewerkschaftsmitglieder, Köln

Sodan, H. (2008): Mindestlöhne und die Grenzen der Verfassung, in: Süddeutsche Zeitung vom 12.7.2008, S.18

Thüsing, G./Lembke, M. (2007): Zeitarbeit im Spannungsfeld von Dienstleistungsfreiheit und Tarifautonomie, in: Zeitschrift für Arbeitsrecht 1, S. $87-125$

Ulber, D./Strauß, S. (2008): Differenzierungsklauseln im Licht der neueren Rechtsprechung zur Koalitionsfreiheit, in: Der Betrieb 36, S. 19701974

Zachert, U. (2004): Tarifvertrag, Günstigkeitsprinzip und Verfassungsrecht, in: Arbeit und Recht 4, S. 121-129

Zachert, U. (2007): Gedanken zur Vertragsfreiheit im Arbeitsrecht aus rechtsvergleichender Sicht, in: Festschrift Richardi, München, S. 11111117

Zachert, U. (2008): Die Tarifautonomie wird wieder entdeckt, in: Süddeutsche Zeitung vom 26. 8. 2008, S. 18

Zachert, U. (2009): Der Streik der Lokführer und die Phantasie der Juristen, in: Liber Amicorum Wendeling-Schröder, Baden-Baden, S. 23-27 